\title{
Kopfschmerz bei Medikamentenübergebrauch
}

\author{
Carl D. Reimers \\ C) Springer-Verlag Berlin Heidelberg 2017 \\ C.D. Reimers, W. Paulus, B.J. Steinhoff (Hrsg.), Patienteninformationen Neurologie - Empfehlungen für Ärzte, \\ DOI 10.1007/978-3-662-53236-2_21
}

?

Was versteht man unter einem Kopfschmerz bei

Medikamentenübergebrauch?

Kopfschmerzen können sich paradox verschlimmern, wenn zu oft Kopfschmerzmittel eingenommen werden. Es kommt zu einer Zunahme der Attackenhäufigkeit und -dauer durch den Gebrauch von Analgetika wie Azetylsalizylsäure, Ibuprofen oder auch Triptanen wegen der Kopfschmerzen. Mit anderen Worten: Der Betroffene mit Kopfschmerzen nimmt häufig Kopfschmerzmittel ein und verursacht dadurch unabsichtlich eine Verstärkung der Kopfschmerzen, die er eigentlich mit den Medikamenten bessern will.

? Wann verursachen Kopfschmerzmedikamente Kopfschmerzen?

Man geht davon aus, dass die Einnahme von Analgetika (Schmerzmittel) oder Triptanen (Medikamente gegen Migräne) selbst wiederum Kopfschmerzen verursachen können, wenn man diese Medikamente an mindestens 15 Tagen pro Monat einnimmt. Die Zahl der Medikamente spielt dabei eine geringere Rolle als die Zahl der Einnahmetage. Der Medikamentenübergebrauch besteht meist über ein Jahr, wobei die Triptane offenbar am raschesten und mit der geringsten Zahl an Einzeldosen pro Monat zu einem Medikamentenübergebrauchskopfschmerz führen. Daher sollte die Zahl der Einnahmetage möglichst 10 nicht überschreiten.

?

Wie äußert sich der Kopfschmerz bei Medikamentenübergebrauch?

Meist ändert sich der Schmerzcharakter wenig gegenüber dem Kopfschmerz, wegen dessen die Medikamente ursprünglich eingenommen wurden. Auch die eventuelle Begleitsymptomatik wie Übelkeit, Erbrechen, Licht- oder Geräuschempfindlichkeit bleibt bei Übergebrauch von Triptanen erhalten. Schmerzmittel wie Ibuprofen, Paracetamol, Azetylsalizylsäure und Ähnliche führen eher zu einem dumpf-drückenden Schmerz. Das macht auch die Diagnose eines Kopfschmerzes durch Medikamentenübergebrauch so schwierig. Man kann ihn nur vermuten, wenn der ursprüngliche Kopfschmerz immer häufiger wird und länger anhält und über eine regelmäßige Medikamenteneinnahme berichtet wird. Diese wird jedoch oft unterschätzt oder verharmlost. In einem Kalender können die Kopfschmerztage und die Medikamenteneinnahme aufgezeichnet werden. Das kann für die Beratung sehr hilfreich sein.

Frauen sind 3- bis 4-mal häufiger betroffen als Männer. Das mittlere Lebensalter überwiegt. Man schätzt, dass weltweit 3-4 \% der Menschen an chronischen Kopfschmerzen leiden, davon etwa jeder Dritte aufgrund eines Medikamentenübergerbrauchs.

? Wie behandelt man den

Medikamentenübergebrauchskopfschmerz?

Die Medikamente, die den mutmaßlichen Medikamentenübergebrauchskopfschmerz verursacht haben, sollen zügig abgesetzt werden. Die Entzugsbehandlung kann ambulant oder stationär vorgenommen werden, in Abhängigkeit von den zu entziehenden Medikamenten, der Dauer des Übergebrauchs, psychischen Begleiterkrankungen und familiärer Unterstützung. Kommt es durch den Entzug (meist nur 2-6 Tage) zu vermehrten Kopfschmerzen, so werden bei starker Ausprägung nichtsteroidale Antiphlogistika (Naproxen), Azetylsalizylsäure-Infusionen oder auch eine mehrtägige Glukokortikoidbehandlung („Kortison“) eingesetzt. Eventuelle Übelkeit und Erbrechen lassen sich mit Metoclopramid oder Domperidon beherrschen. Günstig ist es, gleichzeitig mit dem Entzug eine prophylaktische Behandlung des ursprünglichen Kopfschmerzes einzuleiten. 